\title{
Relaciones entre la crianza y factores protectores o de riesgo, antes y después de una intervención para padres ${ }^{1}$
}

\section{Relationships between parenting and risk or protective factors, before and after a parenting intervention}

\author{
Pedro Solís-Cámara ${ }^{2} \quad$ Yolanda Medina Cuevas \\ Marysela Díaz Romero \\ Universidad de Guadalajara
}

(Rec: marzo 2014 - Acep: junio 2014)

\begin{abstract}
Resumen
Los objetivos del estudio fueron: identificar las relaciones entre la crianza y factores que pueden ejercer influencia protectora o de riesgo para la socialización de los niños y analizar los cambios que fomenta una intervención en crianza. La intervención elegida (Paternidad Estrella), fomenta expectativas justas, y disciplina y crianza positivas; los factores incluidos fueron comportamientos pro sociales de los niños, su desarrollo psicosocial y sus comportamientos problema; así como con el estrés y el coraje/agresión de los padres. Participaron padres con un niño de 3 a 5 años de edad, distribuidos en un grupo experimental, con niños con problemas de conducta $(n=30)$, y uno control $(n=30)$. Los resultados confirman que las intervenciones tempranas modifican problemas de comportamiento; además, mostraron que las expectativas y las prácticas disciplinarias de los padres están relacionadas con los comportamientos pro sociales, el desarrollo y los comportamientos problema de los niños, y con el estrés y el coraje/agresión de los padres. Los resultados se discuten en términos de los esfuerzos por aclarar qué factores de influencia en la crianza pueden ser modificados exitosamente por programas de intervención, y aportan la identificación de componentes específicos que pueden ser incluidos en otros programas.

Palabras clave: crianza, comportamiento problema, desarrollo, estrés, intervención.
\end{abstract}

\begin{abstract}
The objectives of this study were to identify the relationships between parenting and risk factors and protective factors that exert influence on children's socialization process, and to analyze changes in the relationships as are promoted by a programme for parents of young children. The dimensions fostered by the chosen intervention are fair expectations, and discipline and nurturing practices; factors chosen were children pro social behaviors, psycho-social development, behavior problems, as well as anger-aggression and stress from parents. Parents of young children (3 to 5 yrs. old) with behavior problems were assigned to an experimental group $(n=30)$ and parents of children without behavior problems were assigned to a control group $(n=30)$. Results support previous research indicating the importance of early interventions for treatment of behavior problems. Moreover, results allowed the identification of parents' expectations and discipline practices as two components related to children pro social behaviors, psycho-social development, behavior problems, as well as anger-aggression and stress from parents. Results are discussed in terms of present efforts for identifying which factors may be successfully modified, and demonstrate the benefits of specific parenting dimensions which may be adopted by other parenting programs.
\end{abstract}

Keywords: development, interventions, parenting, stress, behavior problems.

\footnotetext{
Esta investigación es resultado del "Proyecto a tres años intervención en crianza y prevención" aprobado y apoyado académica y financieramente por PROMEP-SEP/103.5/09/3912 y la Universidad de Guadalajara, México.

2 Correspondencia a: Pedro Solís-Cámara, Centro de Investigación en Conducta y Cognición Comparada. Centro Universitario de la Ciénega, Universidad de Guadalajara. Av. Universidad \#1115, Lindavista, Ocotlán, Jalisco. México. Código Postal 47820. Teléfono: (52-392) 92 5 94 00. Correo electrónico: psolis@mexis.com.

Agradecimientos: este proyecto fue posible gracias a los apoyos recibidos de PROMEP-SEP/103.5/09/3912, y de la Universidad de Guadalajara. Los autores agradecen también a los estudiantes de psicología, Diana E. Cortes Martínez, Martha Aguirre y Arantxa Martínez, su participación en la calificación de los autoreportes.
} 


\section{Introducción}

En las últimas décadas, los estudios sobre los programas de entrenamiento para padres han acumulado evidencias a favor de sus beneficios sobre el desarrollo psicológico de los niños (Karoly et al., 1998) y también en el estatus psicológico parental, particularmente en el de las madres (e.g., Barlow, Coren \& Stewart-Brown, 2003). Los programas de entrenamiento parental o de intervención en crianza son operacionalizados como estrategias de prevención y/o corrección de problemas de comportamiento externalizadores (e.g., conductas agresivas, desafiantes, etc.) e internalizadores (e.g., ansiedad, aislamiento, temperamento difícil, etc.), tanto en preescolares como en niños escolares de diferentes edades (Levac, McCay, Merka \& Reddon-D'Arcy, 2008; Sanders, Markie-Dadds \& Turner, 2003; Silva \& Randall, 2005; Solís-Cámara, Fox \& Nicholson, 2000).

Por otra parte, los estudios sobre crianza han identificado múltiples factores de influencia en la interacción recíproca padres-niños y han permitido distinguir entre factores protectores para el desarrollo adecuado de los niños (e.g., rol paterno positivo, manejo adecuado del coraje) y factores de riesgo (e.g., NSE bajo, disciplina severa e inconsistente, estrés). Se reconoce que identificar los factores que anteceden, mantienen o favorecen efectos positivos o negativos en las relaciones padresniños es fundamental para el desarrollo de programas preventivos y de intervención (Degnan, Almas \& Fox, 2010). Y, de hecho, un interés actual se centra en diseñar intervenciones que puedan tener un impacto positivo en varios factores de riesgo que predicen socialización inadecuada o pobre en los niños. Por ejemplo, en Inglaterra, Scott et al. (2010) diseñaron una intervención dirigida a modificar cuatro factores de riesgo: crianza ineficiente, conductas problema, síntomas de TDA-H y pobre habilidad de lectura en 112 familias con niños de cinco años de edad al inicio del estudio. La intervención consistió en el programa para padres "Los Años Increíbles" y el programa para lectura SPOKES (por sus siglas en inglés). Los resultados muestran que los padres del grupo control practicaron con sus niños el juego, los halagos, las recompensas y el tiempo fuera, más a menudo que los padres de los grupos controles y practicaron menos la disciplina severa. En cuanto a los niños del grupo de intervención, ellos redujeron de manera significativa $(\mathrm{p}<.05)$, los problemas de conducta, el desorden oposicional-desafiante, los síntomas de TDA-H, y mejoraron en seis meses su edad de lectura.

De manera semejante, en México Solís-Cámara, Covarrubias, Díaz y Rivera (2004) realizaron una intervención con diseño experimental y con padres y madres que trabajaban y tenían un niño(a) de tres a cinco años de edad. Después del entrenamiento, en comparación con un grupo control, el grupo experimental mostró un funcionamiento global familiar más adecuado, los comportamientos verbales y físicos positivos de los padres y sus niños aumentaron, y, en los niños los comportamientos físicos negativos disminuyeron de manera significativa. Además, los cambios en los autoreportes contestados por los padres fueron apoyados por observaciones en vivo, donde los cambios de los padres incluyeron más comportamientos positivos hacia sus niños, tales como: halagos, tocar y abrazar; y menos comportamientos negativos como gritos y golpes. Y, en cuanto a los niños, los comportamientos después del entrenamiento incluían más cooperación y caricias así como menos gritos y pataleos en el suelo. En la fase de seguimiento el $90 \%$ de las familias se ubicaron en la categoría de familias funcionales, donde los padres se percibían a sí mismos menos estresados y con menos coraje y agresión.

Ambos estudios revisados aquí tienen más en común de lo que aparentan. De acuerdo con Weymouth (2010) los programas que utilizaron Scott et al. (2010) y Solís-Cámara, et al. (2004) están entre los más reconocidos en los países de habla inglesa para la reducción de comportamiento agresivo; en el primer caso se trata del programa The Incredible Years (i.e., Los Años Increíbles; Levac et al., 2008) y en el segundo del programa STAR Parenting (Paternidad Estrella; Solís-Cámara et al., 2000). Weymouth (2010) señala en su estudio que estos programas, así como el Parents Raising Safe Kids Program (Padres Criando Niños Seguros; Silva \& Randall, 2005), y el The Triple P Positive Parenting Program (El Programa de Crianza Positiva Triple-P; Sanders et al., 2003), están basados en la teoría del aprendizaje social, todos son estándarizados (i.e., que siguen un Manual para una enseñanza estructurada) y son exitosos para educar a los padres sobre los factores de riesgo en la crianza, incluyendo el abuso infantil. Además agrega que cada uno de estos programas forma parte de la literatura especializada que ha reportado éxitos como métodos de prevención e intervención con padres en riesgo.

En suma, estos programas representan avances porque modifican factores de riesgo para la socialización de los niños. Sin embargo, se ha prestado poca atención a los contenidos específicos de estos programas y cómo afectan a los factores de riesgo para la socialización de los niños. Para conocer estos efectos de los programas se requiere estudiar las relaciones 
entre dimensiones de la crianza y factores, no solo de riesgo si no también protectores. Continuar dilucidando qué "cosas" se hacen en un programa parental y cómo éstas disminuyen los efectos negativos de los factores de riesgo o aumentan los efectos positivos de los factores protectores parece relevante para desarrollar mejores programas de prevención e intervención. Los autores de este estudio creemos que una opción para valorar lo acertado de estas ideas consiste en analizar las relaciones entre dimensiones de la crianza y factores de riesgo o protectores. De hecho, se ha señalado la necesidad de identificar más minuciosamente las influencias entre factores y contenidos específicos de programas de intervención/prevención temprana para mejorar nuestro conocimiento (Degnan et al., 2010). Por ejemplo, si se quiere prevenir la desnutrición, se pueden incluir contenidos de estimulación temprana, la cual ha sido identificada como un factor protector ante la desnutrición (Grantham-McGregor et al., 2007).

Además, existen evidencias de que la tasa de prevalencia de problemas de comportamiento internalizadores muestran, en general, un incremento durante la niñez y adolescencia (Bongers, Koot, van der Ende \& Verhulst, 2003) y la intervención temprana, como estrategia preventiva, ha demostrado tener más beneficios que las estrategias correctivas dirigidas a adolescentes que presentan problemas severos de comportamiento (Dishion \& Patterson, 1992; Hensen \& Jones, 2010; Lochman \& Wells, 2003). En cuanto al programa, se eligió a STAR Parenting (Solís-Cámara et al., 2000) en su adaptación al español, conocido como Paternidad Estrella: Un enfoque positivo y práctico para la paternidad eficiente (Solís-Cámara, Díaz, Fox \& Fox, 2002), para analizar relaciones entre dimensiones de crianza $\mathrm{y}$ factores de riesgo o protectores.

Para lograr la intención de buscar las relaciones mencionadas, es necesario revisar en esta sección las características más sobresalientes del programa. Además es necesario identificar las dimensiones de crianza a las cuales va dirigido y los estudios de efectos sobre factores protectores y de riesgo. El programa fue desarrollado con fundamento en las teorías del desarrollo y cognitivo-conductual para educar a los padres en la formación de niños pequeños (Fox \& Fox, 1992). El programa se divide en cuatro secciones y en dos subprogramas, uno dirigido a padres de infantes y otro a padres de preescolares. El programa puede impartirse en inglés o en español y se enseña en cuatro sesiones de dos y media horas cada una; aunque existen variantes para enseñarlo en ocho sesiones de hora y media. Además se dejan tareas y se ofrecen audio-grabaciones con información y ejemplos adicionales de la aplicación de las técnicas para resolver problemas propios de la crianza (Nicholson, Anderson, Fox \& Brenner, 2002).

Los contenidos del programa enseñan a los padres a hacer alto y pensar antes de actuar con sus niños, desarrollando en los padres autocontrol y manejo del coraje. Estrategias como la de alto y pensar cuentan con evidencias sólidas de su éxito para el control del coraje en los padres (Fetsch, Yang \& Pettit, 2008). Posteriormente enseñan a los padres a preguntarse a sí mismos si sus expectativas acerca de sus niños son justas; es decir, los padres aprenden acerca del desarrollo infantil para poder establecer expectativas sobre lo que sería justo pedir a un niño. Esta estrategia está fundamentada en las evidencias que indican que los padres en general tienen un pobre conocimiento sobre el desarrollo de sus niños pequeños (Ertem et al., 2007) y que existe relación entre pobre conocimiento del desarrollo infantil y las conductas disciplinarias severas de los padres (Fulton, Murphy \& Anderson, 1991). Y las expectativas que se forman los padres con ese conocimiento se ha visto que es fundamental para observar resultados en los niños (Smyth, Whelan, McCoy, Quail \& Doyle, 2010).

Finalmente las características del programa incluyen enseñar a los padres a responder utilizando técnicas disciplinarias y de crianza positivas. Múltiples estudios (Fox, Anderson, Fox \& Rodríguez, 1991; Nicholson et al., 2002; Nicholson, Brenner \& Fox, 1999; Nicholson, Janz \& Fox, 1998; Solís-Cámara et al., 2000; SolísCámara et al., 2004) a lo largo de dos décadas han indicado que la mayoría de los padres utilizan el castigo físico y verbal como técnica disciplinaria y han demostrado que los padres que reciben el entrenamiento, en comparación con grupos controles, disminuyen el uso del castigo, las expectativas irreales o injustas y el coraje; al mismo tiempo se incrementan las interacciones positivas padres-niños y en los niños se reportan menor número de problemas de comportamiento. Las características del programa Paternidad Estrella son flexibles (Nicholson et al., 2002) y de acuerdo a varios estudios (Cardona, Nicholson \& Fox, 2000; Solís-Cámara \& Díaz, 2003) permiten ajustes para cumplir necesidades de comunidades diversas; no sólo va dirigido a niños con problemas de comportamiento y está abierto a cualquiera que tenga contacto con los niños pequeños y no sólo para los padres.

El presente estudio se concentra en padres de niños pequeños, por la relevancia preventiva que representa trabajar con niños de edad preescolar (ver por ejemplo: Hansen \& Jones, 2010). 
El primer objetivo de este estudio es el de identificar las relaciones entre las dimensiones de la crianza, que se argumenta que el programa Paternidad Estrella fomenta, $y$ algunos factores que pueden ejercer influencia protectora o de riesgo para la socialización adecuada de los niños pequeños. Como se ha podido notar el programa Paternidad Estrella le da especial importancia a tres dimensiones de crianza: las expectativas del desarrollo y la disciplina y la crianza positivas. Con la intención de valorar estas dimensiones se eligió el instrumento creado por los autores del programa Paternidad Estrella (Solís-Cámara, 2007): la Escala de Comportamientos para Madres y Padres de Niños Pequeños (ECMP), que valora precisamente esas tres dimensiones de la crianza. En cuanto a los factores de influencia sobre la crianza, se incluyeron medidas de los comportamientos pro sociales de los niños (e.g., juega bien con otros niños), de su desarrollo psicosocial (e.g., se ve asustado, nervioso o temeroso ante situaciones que otros niños de su edad enfrentan sin dificultad), de sus comportamientos negativos (e.g., se queja o llora), de sus comportamientos problema y su intensidad (e.g., actúa desafiante cuando se le pide algo), y del estrés (i.e., temperamento difícil) asignado al niño por sus padres (e.g., mi niño parece que no sonríe tanto como la mayoría).

Como factores de influencia en los padres se eligieron medidas del estrés propio de los padres (e.g., cuando voy a fiestas, usualmente sé que no me divertiré), del estrés percibido en la interacción con su niña o niño (e.g., he visto que el lograr que mi niño haga algo o deje de hacer alguna cosa es mucho más difícil de lo que esperaba), del coraje/agresión (e.g., cuando pierdo la cabeza, amenazo o maldigo). Debido a que los estudios revisados con los programas de intervención argumentan cambios en los factores de influencia mencionados, se eligieron los mismos instrumentos utilizados en esos estudios (e.g., Solís-Cámara et al., 2004); esto, en un esfuerzo por lograr la replicación de los hallazgos anteriores y agregar conocimiento al presentar relaciones específicas entre lo enseñado en el programa de intervención (e.g., expectativas justas de los padres) y factores de influencia (e.g., comportamiento problema).

Aunque las variables socio demográficas no se incluyeron como factores de influencia, se recolectó información que se reconoce (Izzedin \& Pachajoa, 2009; Solís-Cámara \& Díaz, 2002) que puede ejercer influencia en la crianza, particularmente la escolaridad y los ingresos económicos; esto con la intención de comparar sus distribuciones entre grupos.
El segundo objetivo de este estudio es el de analizar los cambios, que se argumenta que el programa Paternidad Estrella fomenta, en las relaciones encontradas en un grupo de padres con niños preescolares con problemas de conducta expuestos a la intervención y comparar si hubo cambios en las relaciones encontradas, tres meses después, contra un grupo de padres de niños sin problemas de conducta y no expuestos a la intervención. Se consideró relevante explorar las relaciones mencionadas en grupos de padres con niños con problemas de conducta o sin problemas de conducta, porque esto ofrecería datos más ricos sobre qué relaciones cambian después de una intervención.

En suma, este estudio tiene como propósito identificar si las expectativas que tienen los padres sobre el desarrollo de sus niños y sus prácticas disciplinarias y de crianza se relacionan con el comportamiento prosocial, el desarrollo psicosocial, los comportamientos problema y el estrés de los niños, y con el estrés y el coraje/agresión de los padres. Otro propósito del estudio es analizar si las relaciones halladas se modifican después de que los padres participan en un programa de intervención para padres.

\section{Método}

\section{Participantes}

Se trata de un estudio con diseño cuasi-experimental. El diseño consistió en un grupo experimental (GE) y un grupo control (GC), donde las mismas variables dependientes fueron repetidas para los dos grupos, siendo la variable independiente: grupo expuesto al programa de crianza versus GC. La selección de la muestra fue no probabilística e intencional; cualquier mamá, papá, o ambos $(n=60)$ con al menos un niño (i.e., niña o varón) de 3 a 5 años de edad, con o sin problemas de conducta y sin tratamiento psicológico, según el personal escolar, e inscrito en alguna de las instituciones, pudo ser incluido en este proyecto. Al ser identificados todos los padres, éstos fueron asignados al GE si sus niños habían sido identificados con anterioridad con la etiqueta de "niño problema" por ser de difícil manejo según el personal de las estancias. El otro grupo quedó formado por niños supuestamente no problemáticos. Los padres fueron reclutados en cinco estancias públicas donde asistían sus niños; las estancias estaban ubicadas en diferentes zonas de la $\mathrm{Cd}$. de Guadalajara en México. Los padres del GE $(n=20$ mamás y 10 
papás) completaron las evaluaciones de pretest o preentrenamiento, el programa de crianza (8 semanas), y las evaluaciones de postest. Los del GC $(n=21$ mamás y 9 papás) completaron las evaluaciones de pretest, que fueron repetidas a las 8 semanas (pretest-II).

\section{Instrumentos}

Información de la Familia. Cuestionario que pide la información básica de la familia. Edades de padres e hijos, escolaridad y ocupación de cada miembro de la familia e ingresos económicos familiares.

Escala de Comportamientos para Madres y Padres de Niños Pequeños (ECMP). La ECMP (Solís-Cámara, 2007) consta de 99 reactivos calificados con una escala Likert de cuatro puntos y distribuidos en tres subescalas: Expectativas que tienen los padres acerca del desarrollo de sus niños; calificaciones muy altas indican que los padres esperan que su niño realice tareas o actividades muy difíciles de lograr por niños de esa edad y calificaciones bajas indican que no se exige al niño que realice tareas que un niño de esa edad puede lograr con facilidad. Las prácticas disciplinarias de los padres ante los comportamientos de los niños; altas calificaciones indican disciplinas verbal y física severas. Crianza: mide específicamente los comportamientos de los padres para promover el desarrollo psicológico de los niños; altas calificaciones indican que los padres ofrecen actividades y apoyos positivos. La confiabilidad alfa total de la ECMP es de .95 .

Inventario Eyberg del Comportamiento del Niño (IECN). El IECN (Eyberg \& Ross, 1978) mide los problemas de comportamiento comunes en los niños de 2 a 16 años de edad. Se ha demostrado que esta escala discrimina entre niños con y sin problemas. Los padres califican la frecuencia de cada conducta en una escala del 1 (nunca) hasta el 7 (siempre); la calificación total que resulta es llamada de intensidad (rango: 36253). Se pide también que los padres califiquen con una respuesta de sí o no, en cada reactivo, si cada una de las conductas es o no un problema para su niño actualmente resultando en una calificación problema (rango: 136). Se han publicado confiabilidades alfa desde .67 hasta .93 (Burns \& Patterson, 2000).

Cuestionario de Valoración del Comportamiento $(C V C)$. El CVC fue tomado de un estudio para la valoración de niños preescolares con problemas significativos de comportamiento y emocionales (Solís-Cámara \& Díaz, 2002); en él se indica que la confiabilidad entre los observadores calificadores para el instrumento original iban de .77 a .94. Para este estudio, se conservaron 12 problemas de conducta comunes en niños pequeños (e.g., berrinches, ir al baño, irse a dormir), pero se agregaron 14 reactivos valorando el comportamiento pro social de los pequeños (e.g., juega bien con otros niños, sonríe/ríe). Utiliza una escala de cuatro puntos (de $4=$ casi siempre/siempre hasta $1=$ casi nunca/nunca).

Escala de Salud del Niño (ESN). La ESN (Berganza, Cazali, Gaitán \& Mezzich, 1996). Es una medida breve que consta de 12 reactivos para valorar la percepción que del niño se hace el adulto en la dimensión de desadaptación psicosocial, basada en la taxonomía diagnóstica internacional de población infantil, e incluye reactivos sobre la salud, conducta, emociones, social y académico. Los reactivos son calificados utilizando una escala de cuatro puntos $(0=$ nada o casi nada/nunca, $1=$ poco o algunas veces, y $2=$ bastante/ frecuentemente, 3 = mucho, siempre/o casi siempre). La ESN fue validada en Guatemala, con sensibilidad de 89.0, y especificidad de 87.7 para 5 puntos como criterio de corte para indicar una probabilidad justa de disfunción en el desarrollo psicosocial.

Cuestionario Breve de Coraje y Agresión (CBCA). El CBCA (Maiuro, Vitaliano \& Cahn, 1987) es una medida de seis reactivos para valorar los niveles de coraje y agresión. Los reactivos son calificados en una escala del 0 -extremadamente improbable a 4 -muy probable, con un total de calificaciones del 0 al 24 (la calificación de corte en el 9 indica una probabilidad justa de descontrol por coraje o agresión). La confiabilidad alfa total del CBCA es de .82 .

Índice de Estrés Paterno (IEP). El IEP (Abidin, 1995) es un instrumento de 36 reactivos que miden el estrés en tres áreas: estrés paterno personal, estrés relacionado con la interacción del padre y el niño, y el estrés que resulta de las características únicas del niño. Los reactivos se califican en una escala tipo Likert que va de totalmente de acuerdo hasta totalmente en desacuerdo. Múltiples estudios apoyan la confiabilidad y estabilidad temporal de este instrumento (Ostberg \& Hagekull, 2000).

\section{Procedimientos}

Todos los padres completaron una forma de consentimiento aprobada por las instituciones; además, el estudio cumple con la Declaración de Helsinki de 2004 y el Código Ético del Psicólogo (Sociedad Mexicana de Psicología, 2007), en particular con los artículos, del 31 al 35, referentes a la investigación con participantes humanos; el estudio cumple también con las normas de la Ley General de Salud de México sin implicar ningún riesgo para la salud. 
Programa de crianza. El programa de Paternidad Estrella se impartió en 8 sesiones de 1.5 horas, una cada semana. En el programa para padres (Solís-Cámara et al., 2002) se les enseñó las etapas del desarrollo, el establecimiento de expectativas justas sobre el desarrollo de sus niños, técnicas disciplinarias (ignorar, sobrecorrección, consecuencias, tiempo fuera) y técnicas de crianza (cómo dar instrucciones, prestar atención, mirando hacia adelante, actividades recreativas, reforzamiento positivo). En todos los segmentos se dejó tarea para alentar a practicar las estrategias cognitivas y las técnicas conductuales. Los padres escucharon audio-grabaciones para reforzar el material de clases y los libros de trabajo para los ejercicios en clase y para las aplicaciones en el hogar. El programa de crianza fue enseñado por educadores certificados con apoyo del Consejo Nacional de Ciencia y Tecnología de México. Está basado en la guía de líderes, libro de 200 páginas ilustrando conceptos y aplicaciones (Solís-Cámara, Díaz, Fox \& Fox, 2001).

Clases de crianza. En cada uno de los sitios se llevaron a cabo juntas de orientación para explicar el proyecto, obtener consentimiento firmado por los padres, etc. Los padres fueron programados para la sesión de pretest con su niño. A los padres asignados al grupo control se les dijo que el inicio de sus clases se demoraría por lo menos 10 semanas; esto se realizó con la intención de evaluar a los padres nuevamente y poder comparar sus calificaciones con las de los padres que habían tomado el entrenamiento. Los padres del grupo experimental fueron entrenados en grupos de 1 a 4 personas e iniciaron el programa la semana siguiente al pretest.

\section{Procedimiento de análisis estadístico}

El análisis de los datos se realizó con el programa Statistica (Stat Soft Ver. 9.1). Se realizaron análisis descriptivos, MANOVAs para identificar los efectos principales, y análisis univariados para confirmar diferencias. También se realizaron MANOVAs de medidas repetidas para valorar los cambios del pre al post entrenamiento. Se realizaron también correlaciones Pearson para valorar las relaciones y su dirección entre variables.

\section{Resultados}

Como se puede observar la distribución de padres casados fue semejante en ambos grupos; sin embargo, en escolaridad, ocupación e ingresos económicos, las familias del grupo control (GC) muestran mayor escolaridad, mejores ocupaciones y mejores ingresos económicos mensuales que las familias del grupo experimental. La edad promedio de los padres (20 mamás, 10 papás) del grupo experimental (GE: $M=35.0, D E$ $=6.2$ ) y de sus niños (14 niñas, 16 varones; $M=3.6$,

Tabla 1.

Distribución porcentual de características socioeconómicas de los grupos.

\begin{tabular}{|c|c|c|c|c|}
\hline & \multicolumn{4}{|c|}{ Grupos } \\
\hline & \multicolumn{2}{|c|}{ Experimental } & \multicolumn{2}{|c|}{ Control } \\
\hline & $\mathrm{n}$ & $\%$ & $\mathrm{n}$ & $\%$ \\
\hline \multicolumn{5}{|l|}{ Estado civil } \\
\hline Casado & 25 & 83 & 27 & 90 \\
\hline Divorciado (o semejante) & 5 & 17 & 3 & 10 \\
\hline \multicolumn{5}{|l|}{ Escolaridad } \\
\hline 12 años (nivel medio) & 20 & 67 & 15 & 50 \\
\hline$>$ de 13 años (universidad) & 10 & 33 & 15 & 50 \\
\hline \multicolumn{5}{|l|}{ Ocupación } \\
\hline Empleados & 18 & 60 & 19 & 63 \\
\hline Profesionistas & 4 & 13 & 7 & 23 \\
\hline Otras (técnicos, obreros) & 8 & 27 & 4 & 14 \\
\hline \multicolumn{5}{|l|}{ Ocupación de la pareja } \\
\hline Empleados & 12 & 40 & 8 & 27 \\
\hline Profesionistas & 5 & 17 & 11 & 37 \\
\hline Otras (técnicos, obreros) & 13 & 43 & 11 & 36 \\
\hline \multicolumn{5}{|l|}{ Ingresos económicos } \\
\hline Hasta 10 mil pesos (< de $\$ 1000$ US $)$ & 25 & 83 & 21 & 70 \\
\hline Más de 10 mil pesos & 5 & 17 & 9 & 30 \\
\hline
\end{tabular}


$D E=0.8)$ fue semejante a la de los padres (21 mamás, 9 papás; $M=35.7, D E=6.8)$ y niños del GC (8 niñas, 22 varones; $M=3.8, D E=0.8$ ) (Ver Tabla 1$)$.

Las calificaciones de pretest para el GE $(M=$ $140.4, D E=15.9)$ y para el GC $(M=146.5, D E=$ 18.7) en la dimensión de expectativas no se encontraron diferencias estadísticamente significativas $(F(1,58)=1.81, p=0.18)$, a pesar de que se observa más pobres expectativas en el GE que en el GC. En la Tabla 2 se muestran las medias y desviaciones de las calificaciones de pretest de ambos grupos en todos los factores de influencia. En cuanto a los resultados del MANOVA no se encontraron diferencias significativas entre el GC y el GE $(F(9,50)$
$=0.74, p=0.66)$. Sin embargo, las calificaciones del GE en desarrollo rebasan el punto de corte de 5 , que indica una probabilidad justa de disfunción en el desarrollo psicosocial. Y un ANOVA de las calificaciones de coraje/agresión entre el GC y el GE indicó calificaciones de coraje más altas en el GE $(F(1,58)=3.52, p=.06)$. En la Tabla 2 se presentan también correlaciones de Pearson significativas $(p<.05)$. Se observa que en el GE sólo se encontraron correlaciones significativas indicativas de que pobres expectativas hacia el desarrollo de los niños se relacionan con más problemas de conducta y mayor coraje/agresión de los padres. En el GC, no se encontraron correlaciones significativas.

Tabla 2.

Estadísticas descriptivas y correlaciones entre la dimensión de expectativas y factores de influencia en la crianza de los grupos antes de la intervención.

\begin{tabular}{|c|c|c|c|c|c|c|c|c|}
\hline \multirow[b]{3}{*}{ Dimensión vs. Factor } & \multicolumn{8}{|c|}{ Pretest } \\
\hline & \multicolumn{4}{|c|}{ Grupo Experimental } & \multicolumn{4}{|c|}{ Grupo Control } \\
\hline & $r$ & $p=$ & $M$ & $D E$ & $r$ & $p=$ & $M$ & $D E$ \\
\hline \multicolumn{9}{|l|}{ Expectativas } \\
\hline Comportamiento positivo & .04 & .82 & 40.7 & .32 & .32 & .09 & 43.1 & 5.3 \\
\hline Comportamiento negativo & .28 & .13 & 24.4 & 4.3 & -.07 & .72 & 24.7 & 4.6 \\
\hline Problemas de conducta & -.37 & .04 & 15.5 & 9.7 & -.02 & .92 & 13.1 & 7.7 \\
\hline Intensidad de los problemas & -.33 & .07 & 119.2 & 34.7 & -.20 & .28 & 111.1 & 27.7 \\
\hline Desarrollo & -.34 & .06 & 5.5 & 4.7 & -.12 & .54 & 4.8 & 3.7 \\
\hline Estrés padres & -.31 & .09 & 28.1 & 6.7 & .08 & .68 & 26.9 & 7.8 \\
\hline Estrés interacción & -.26 & .16 & 23.1 & 7.6 & -.23 & .22 & 20.9 & 6.7 \\
\hline Estrés en el niño & -.29 & .11 & 29.2 & 7.3 & -.17 & .37 & 27.2 & 7.9 \\
\hline Coraje/agresión & -.43 & .01 & 10.2 & 3.4 & -.26 & .16 & 8.3 & 4.3 \\
\hline
\end{tabular}

Los resultados del MANOVA de medidas repetidas para las tres dimensiones de crianza mostró diferencias estadísticamente significativas en las calificaciones del pre a postest del GE $(F(3,27)=25.9, p<0.001)$. En el caso de la dimensión de expectativas, el análisis univariado confirmó los cambios $(F(1,29)=12.3, p<0.01)$ del pre al postest $(M=148.9, D E=15.8)$. En cuanto al GC, sus calificaciones del pre al pretest-II $(M=147.4, D E=$ 19.6) fueron muy semejantes, como sería de esperar. Las calificaciones postest del GE se incrementaron significativamente y fueron más altas que las de pretest-II del GC, pero la comparación entre ellas no fue estadísticamente significativa $(F(1,58)=0.12, p=0.74)$.

En la Tabla 3 se muestran las medias y desviaciones de las calificaciones de postest del GE y de pretest-II del GC en todos los factores de influencia y sus correlaciones con la dimensión de expectativas. Los resultados del MANOVA de medidas repetidas para los factores de influencia del GE mostraron que había efectos principales significativos $(F(9,21)=$ $3.19, p<.01)$; los efectos se confirmaron con análisis univariados para todas las variables que, como se puede observar al comparar las Tablas 2 y 3 , se debieron a cambios en todos los factores; siendo todos significativos (de $p<.01$, por lo menos; debido a su extensión no se presentan los ANOVAs). En contraste, no se observaron cambios en el GC $(F(9,21)=$ $1.44, p=0.23$ ), como sería de esperar. Para el GE se observa en la Tabla 3, que el incremento sustancial, más no excesivo, en las expectativas se relacionó con menor número de comportamientos negativos y de problemas de conducta, pero sobretodo con menor percepción sobre la intensidad de los problemas y del estrés asignados al niño, que muestran correlaciones de nivel moderado a fuerte. En cambio en el GC no se encontraron correlaciones significativas. 
Tabla 3.

Estadísticas descriptivas y correlaciones entre la dimensión de expectativas y factores de influencia en la crianza durante el postest y el pretest-II.

\begin{tabular}{|c|c|c|c|c|c|c|c|c|}
\hline \multirow[b]{3}{*}{ Dimensión vs. Factor } & \multicolumn{4}{|c|}{ Grupo Experimental } & \multicolumn{4}{|c|}{ Grupo Control } \\
\hline & \multicolumn{4}{|c|}{ Postest } & \multicolumn{4}{|c|}{ Pretest-II } \\
\hline & $r$ & $p=$ & $M$ & $D E$ & $r$ & $p=$ & $M$ & $D E$ \\
\hline \multicolumn{9}{|l|}{ Expectativas } \\
\hline Comportamiento positivo & -.03 & .87 & 44.7 & 4.5 & .29 & .11 & 42.5 & 5.5 \\
\hline Comportamiento negativo & -.41 & .03 & 20.2 & 3.5 & -.18 & .33 & 23.7 & 4.1 \\
\hline Problemas de conducta & -.35 & .05 & 10.6 & 6.6 & -.32 & .09 & 13.6 & 8.4 \\
\hline Intensidad de los problemas & -.61 & .001 & 95.6 & 28.3 & -.07 & .73 & 106.3 & 25.4 \\
\hline Desarrollo & -.20 & .28 & 3.2 & 3.5 & -.17 & .35 & 4.6 & 3.7 \\
\hline Estrés padres & -.23 & .21 & 22.6 & 7.6 & .07 & .69 & 25.1 & 6.3 \\
\hline Estrés interacción & -.25 & .19 & 19.6 & 7.5 & -.15 & .44 & 21.1 & 7.4 \\
\hline Estrés en el niño & -.46 & .01 & 23.7 & 7.1 & -.18 & .34 & 27.3 & 7.4 \\
\hline Coraje/agresión & -.30 & .10 & 7.2 & 4.5 & .29 & .12 & 8.5 & 3.9 \\
\hline
\end{tabular}

Las calificaciones de pretest para el GE $(M=46.2$, $D E=9.4)$ y para el GC $(M=46.0, D E=9.9)$ en la dimensión de prácticas disciplinarias fueron prácticamente idénticas $(F(1,58)=0.009, p=0.92)$. En la Tabla 4 se muestran las correlaciones entre la dimensión de prácticas disciplinarias y todos los factores de influencia; las medias y desviaciones de los factores fueron presentadas antes (Tabla 2). Se observa que todas las correlaciones entre disciplina y los factores fueron positivas y significativas en el GE, con excepción de comportamientos positivos; estas correlaciones indican que prácticas disciplinarias severas se relacionan con más comportamientos negativos, más conductas problema y mayor intensidad de las mismas, mayor estrés en los padres, en la interacción y en el niño y, también, más coraje. En cuanto a la escala de desarrollo de los niños, mayores calificaciones indican más pobre desarrollo, por ello la correlación encontrada indica que menor desarrollo de los niños se relaciona con prácticas disciplinarias más severas. En el GC prácticamente se encontraron los mismos resultados, con excepción de la correlación de prácticas disciplinarias y problemas de conducta.

Tabla 4.

Correlaciones entre las prácticas disciplinarias y factores de influencia en la crianza para los grupos antes de la intervención.

\begin{tabular}{|c|c|c|c|c|}
\hline \multirow[b]{3}{*}{ Dimensión vs. Factor } & \multicolumn{4}{|c|}{ Pretest } \\
\hline & \multicolumn{2}{|c|}{ Grupo Experimental } & \multicolumn{2}{|c|}{ Grupo Control } \\
\hline & $\mathrm{r}$ & $\mathrm{p}=$ & $\mathrm{r}$ & $\mathrm{p}=$ \\
\hline \multicolumn{5}{|l|}{ Disciplina } \\
\hline Comportamiento positivo & .03 & .89 & -.27 & .14 \\
\hline Comportamiento negativo & .47 & .009 & .49 & .007 \\
\hline Problemas de conducta & .39 & .03 & .34 & .06 \\
\hline Intensidad de los problemas & .37 & .04 & .43 & .01 \\
\hline Desarrollo & .40 & .02 & .42 & .02 \\
\hline Estrés padres & .35 & .05 & .56 & .001 \\
\hline Estrés interacción & .64 & .000 & .41 & .02 \\
\hline Estrés en el niño & .47 & .009 & .38 & .04 \\
\hline Coraje/agresión & .38 & .04 & .44 & .01 \\
\hline
\end{tabular}


Debido a que en el MANOVA de medidas repetidas, presentado antes para las tres dimensiones consideradas, se encontró efectos principales significativos en las calificaciones de pre a postest del GE, se realizó un análisis univariado para la dimensión de disciplina. El análisis de medidas repetidas confirmó un decremento sustancial de las prácticas disciplinarias severas $(F(1,29)=46.4, p<0.001)$ del pre al postest $(M=35.3, D E=5.1)$. En cuanto al $\mathrm{GC}$, sus calificaciones del pre al pretest-II $(M=46.2, D E=$ 11.2) fueron prácticamente las mismas, como sería de esperar. Debido a que las calificaciones postest del GE disminuyeron significativamente y fueron más bajas que las de pretest-II del GC, la diferencia entre ellas fue estadísticamente significativa $(F(1,58)$ $=23.6, p<0.001$ ).

Tabla 5.

Correlaciones entre prácticas disciplinarias y factores de influencia en la crianza durante el postest y el pretest-II.

\begin{tabular}{lcccc}
\hline & $\begin{array}{c}\text { Grupo Experimental } \\
\text { Postest }\end{array}$ & \multicolumn{2}{c}{$\begin{array}{c}\text { Grupo Control } \\
\text { Pretest-II }\end{array}$} \\
\hline Dimensión vs. Factor & $r$ & $p=$ & $r$ & $p=$ \\
\hline Disciplina & & & & \\
$\quad$ Comportamiento positivo & .00 & .97 & -.02 & .92 \\
Comportamiento negativo & .14 & .47 & .45 & .01 \\
Problemas de conducta & .01 & .94 & .31 & .09 \\
Intensidad de los problemas & .17 & .36 & .46 & .01 \\
Desarrollo & .45 & .01 & .36 & .04 \\
Estrés padres & .25 & .18 & .65 & .000 \\
Estrés interacción & .21 & .27 & .66 & .000 \\
Estrés en el niño & .17 & .37 & .55 & .002 \\
Coraje/agresión & .49 & .005 & .56 & .001 \\
\hline
\end{tabular}

En la Tabla 5 se observa que únicamente dos correlaciones de las encontradas en el pretest, se conservaron en el GE; una indicando que prácticas disciplinarias menos severas se relacionan con mejor desarrollo de los niños y, la otra, indicando que éstas también se relacionan con menor coraje/agresión de los padres. En el GC, en la fase pretest-II encontramos correlaciones significativas y en la misma dirección a las encontradas durante el pretest; pero éstas pasaron de ser correlaciones moderadas a fuertes, en particular para los tipos de estrés.

En cuanto a las prácticas de crianza, las calificaciones del pretest fueron semejantes entre el GE $(M=51.8, D E=7.0)$ y el GC $(M=52.8, D E=7.5)$. En el GE no se encontró una sola correlación significativa. En el GC hubo una sola correlación significativa entre crianza y comportamiento positivo $(r=.46, p=.01)$, indicando que a más prácticas de crianza positivas, más comportamientos positivos en el niño. El resultado del ANOVA de medidas repetidas para el GE del pre al postest $(M=58.2, D E=7.5)$ mostró cambios estadísticamente significativos $(F(1,29)=26.7, p<.001)$, que se suman a los cambios en todos los factores después de la intervención (Tabla 2). En contraste, no se observaron cambios del pre al pretest-II $(M=52.3, D E=7.5)$ en las prácticas de crianza del GC $(F(1,29)=0.12, p=0.72)$, como sería de esperar. Debido a que las calificaciones postest del GE aumentaron significativamente y fueron más altas que las de pretest-II del GC, la comparación entre ellas fue estadísticamente diferente $(F(1,58)=9.27, p<0.01)$.

Por otra parte, se encontró en el GE una sola correlación significativa postest entre prácticas de crianza y comportamiento positivo y ésta fue incluso más fuerte $(r=.52, p=.003)$ que la del GC durante el pretest. Sin embargo, en la fase de pretest-II del GC la correlación de crianza también se hizo más fuerte $(r=.51, p=.004)$.

\section{Discusión}

Los objetivos del presente estudio fueron: identificar las relaciones entre algunas dimensiones de la crianza y algunos factores que pueden ejercer influencia protectora o de riesgo para la socialización de los niños $\mathrm{y}$ analizar los cambios que fomenta un programa de 
intervención en crianza. Se compararon dos grupos con características diferentes: el grupo experimental (GE) que estaba formado por padres con niños con problemas de conducta y expuesto a una intervención en crianza y el grupo control (GC) formado por padres con niños sin problemas de conducta y que no fue expuesto a la intervención. Además, en nuestro medio escolar la asignación de la etiqueta "problemas de conducta" a los niños, es común y tiene repercusiones negativas para el niño y su familia, tal como expulsar al niño de la escuela por considerarlo "problemático".

Por otra parte, es importante notar también que el grupo de padres que no recibió entrenamiento presentaba características sociodemográficas diferentes a las del GE, tales como mayores concentraciones de padres con más años de escolaridad, mejores ocupaciones y mejores ingresos económicos mensuales; lo que indica que el GE tenía, comparativamente, escasos recursos en general. Esto es importante porque desde hace décadas se reconoce que tanto como una tercera parte de la varianza de los problemas de conducta reportados en niños, se pueden atribuir a condiciones medioambientales adversas (Conger, McCarty, Yang, Lahey \& Kropp, 1984) y esas condiciones negativas se han reportado como factores directos de influencia en la crianza (Solís-Cámara \& Díaz, 2002). Por lo tanto, es conveniente tener presente estas características de los grupos al considerar los resultados.

En cuanto a las calificaciones de la dimensión de expectativas en la fase de pretest, no se encontraron diferencias estadísticamente significativas entre el GE y el GC; y tampoco se encontraron diferencias significativas entre los factores estudiados. Estos resultados pueden interpretarse en términos de la inexistencia de relación entre la dimensión de expectativas y los factores analizados. Sin embargo, la observación cuidadosa de las calificaciones de los grupos indicaron que el GE comparado con el GC sí tenía calificaciones más bajas en la dimensión de expectativas y, en cuanto a los factores, también tenía calificaciones más bajas en comportamientos positivos, más altas en conductas problema y en la intensidad de problemas, menor desarrollo, más estrés y sobre todo una tendencia probabilística $(p=06)$ a que los padres presentaran más coraje-agresión de tipo instrumental (i.e., golpes y gritos). Aún con esta observación cuidadosa los resultados no permiten refutar la interpretación anterior. Sin embargo, se debe recordar que se encontraron correlaciones significativas en el pretest solo para el GE, y estas indicaron que bajas expectativas estaban relacionadas con comportamientos problemáticos en los niños (problemas de conducta como: no obedece, se queja, golpea, etc.; Eyberg, Boggs \& Rodriguez, 1992; Eyberg \& Ross, 1978) y en los padres con mayor coraje-agresión. Estos resultados sugieren que sí existe relación entre las expectativas $\mathrm{y}$ algunos de los factores estudiados, pero para apoyar este argumento se requiere estudios con muestras que presenten diferentes "niveles" de expectativas, como son bajas, justas y altas.

Del pretest al postest (GE) o del pretest al pretest-II (GC), se observó un incremento sustancial de las expectativas del grupo entrenado que ahora eran semejantes a las del GC donde permanecieron prácticamente sin cambios. Más importante aún es el hecho de que todos los factores de influencia se modificaron positivamente en el GE pero no en el GC. Las expectativas, ahora más altas de los padres, se pueden interpretar como más justas para la edad de los niños; esto porque las correlaciones de postest indicaron que las expectativas se relacionaban con menos comportamientos negativos (e.g., berrinches, llantos, etc.) y problemas de conducta, pero particularmente con una percepción paterna de menor intensidad de los problemas y menos estrés asignado al niño (e.g., se despierta de mal humor, no bromea ni ríe, etc.). En contraste con estos resultados en el GC no se encontraron correlaciones significativas.

Estos resultados confirman la importancia de incluir la enseñanza del desarrollo infantil, tal como se cubre con el programa Paternidad Estrella; es decir, presentando las conductas propias de niños y niñas por áreas del desarrollo (i.e., motora, socio-emocional, lenguaje y adaptativa) y por grupos de edad (de 1-3 años o de 3 a 5 años), incluyendo ejemplos y ejercicios. Lo que permite a los padres aprender a identificar expectativas bajas (e.g., esperar que un niño de tres años no pueda jugar solo unos cuantos minutos), altas (e.g., esperar que un niño de tres años siempre tome turno durante el juego) o justas para su niño en función de su propio desarrollo (Solís-Cámara et al., 2001).

En cuanto a las calificaciones de pretest de las prácticas disciplinarias, éstas fueron prácticamente iguales entre grupos. Con respecto a las relaciones en el GE, a diferencia de la dimensión de expectativas, en la de prácticas disciplinarias se encontraron correlaciones significativas y positivas con todos los factores de influencia, con excepción de comportamientos positivos. Un patrón semejante de relaciones se encontró en el GC, con excepción de problemas de conducta; es decir, solo en el grupo de "niños problemáticos" se encontró relación entre las prácticas disciplinarias de los padres y los problemas de conducta. En general esto indica que, a más prácticas disciplinarias severas, menor desarrollo 
de los niños, más comportamientos negativos, más conductas problema y mayor intensidad de las mismas, mayor estrés en los padres, en la interacción y en el niño y, también, más coraje-agresión en los padres.

Las prácticas disciplinarias severas disminuyeron de manera importante del pre al postest en el grupo entrenado, pero no del pre al pretest-II en el GC, por lo que la diferencia entre ambos grupos fue significativa. Pero, además, la mayoría de las correlaciones entre la dimensión de disciplina y los factores de influencia perdieron significancia en el GE; y solo se encontraron aquellas indicando que un mejor desarrollo de los niños y menor coraje-agresión de los padres se relacionaba con prácticas menos severas. En contraste, en la fase de pretest-II del GC no solo se encontraron las mismas relaciones de la fase de pretest, si no que las de estrés y coraje-agresión se hicieron aún más fuertes.

¿Cómo explicar las diferentes relaciones en la fase de postest y pretest-II, si las prácticas disciplinarias de ambos grupos eran casi idénticas durante el pretest? Una diferencia que es importante señalar es que las calificaciones pretest de los factores de influencia negativos (e.g., problemas de conducta e intensidad de los mismos y los diferentes tipos de estrés) del GE eran mayores que los del control, aún cuando estas diferencias no fueran significativas. Pero más importante aún es el hecho de que durante el pretest, si se observan las medias aritméticas de desarrollo del niño y de coraje/agresión de los padres del GE (5.5 y 10.2, respectivamente), se notará que ambas rebasaron los puntos de corte (5 y 9), señalados por los autores de esos instrumentos para un diagnóstico significativo (Berganza et al., 1996; Maiuro et al., 1987); en cambio, en el GC las calificaciones estaban cercanas a los puntos de corte ( 4.8 y 8.3 ) y así se conservaron durante el pretest-II (4.6 y 8.5); en contraste, durante la fase de postest del GE, esas calificaciones disminuyeron muy por abajo del punto de corte (3.2 y 7.2). Estos resultados sugieren que el programa de intervención fue muy exitoso, particularmente con la dimensión de prácticas disciplinarias y los factores de desarrollo del niño y de coraje/agresión de los padres.

En contraste con los resultados de las dimensiones de expectativas y disciplina, el cómo se comportan los padres con sus hijos al ofrecerles experiencias positivas; es decir, prácticas de crianza positiva (e.g., "Yo paso al menos una hora al día jugando con mi niño/a, o leyéndole"), no fue igual de relevante en términos de los factores de influencia. Las calificaciones de pretest fueron semejantes entre grupos y no se encontraron correlaciones significativas en el GE; en el GC se encontró una relación indicando que a más prácticas de crianza positiva más comportamientos positivos en los niños; lo cual es congruente con este grupo sin problemas de conducta. Sin embargo, es interesante notar que la intervención modificó de manera importante las prácticas de crianza positiva; al grado de superar en el postest las prácticas del GC de la fase de pretest-II y encontrarse una correlación semejante a la del GC, indicando la relación entre prácticas de crianza y comportamientos positivos en los niños.

Por otra parte, los resultados del presente estudio confirman resultados anteriores con la intervención elegida; ya que a lo largo de dos décadas han reportado que los padres de niños pequeños que reciben el entrenamiento, en comparación con grupos controles, disminuyen el uso de la disciplina severa (i.e., castigo), modifican las expectativas irreales o injustas y aumentan sus prácticas de crianza positiva (Fox et al., 1991; Nicholson et al., 1998; Nicholson et al., 1999; Nicholson et al., 2002; Solís-Cámara et al., 2000; Solís-Cámara et al., 2004). Claramente la investigación actual sobre las intervenciones basadas en las teorías del aprendizaje social indica que éstas pueden implementarse de manera exitosa para aminorar los efectos de factores de riesgo en la crianza (Levac et al., 2008; Sanders et al., 2003; Silva \& Randall, 2005).

Tal como lo señaló Weymouth (2010), el Programa Estrella indica que la reducción del comportamiento problema se logra por el énfasis del programa en alternativas disciplinarias tales como ignorar, tiempo tranquilo y consecuencias naturales. Por otra parte, las estrategias cognitivas de alto y piensa que se implementan a lo largo de todo el programa confirman su utilidad para la reducción del coraje y la agresión instrumental que forma parte de las prácticas tradicionales de castigo, tales como son los golpes y los gritos (Fetsch et al., 2008). Además, la estrategia de pregunta sobre si las expectativas de los padres se establecen en función del propio desarrollo del niño, que también se implementa a lo largo del programa, favorece el establecimiento de expectativas más justas. La investigación contemporánea indica que los padres en general desconocen el desarrollo de sus niños (Ertem et al., 2007) y las expectativas que se forman los padres al adquirir ese conocimiento se considera fundamental para lograr cambios positivos en los niños (Smyth et al., 2010).

Si además se integra el establecimiento de expectativas justas y las alternativas disciplinarias adecuadas, se modifica la relación reportada en otras investigaciones entre un pobre conocimiento del desarrollo infantil y conductas disciplinarias severas de los padres (Fulton et al., 
1991). Además, la estrategia de Respuesta, que sustituye las reacciones emocionales por respuestas bien pensadas, favorece también la reducción de prácticas disciplinarias severas en la intervención llevada a cabo en este estudio (Solís-Cámara et al., 2001; Solís-Cámara et al., 2002).

En suma, los resultados de este estudio en el contexto de la formación de los niños, permiten establecer la hipótesis de que dos dimensiones en las experiencias de los niños pueden modular los factores de influencia en la crianza, al incrementar los efectos positivos y al lograr disminuciones de los efectos adversos: 1) lo que los padres esperan de sus niños (expectativas de los padres, por ejemplo: mi niño(a) debe ser capaz de alimentarse por sí mismo(a), que se refieren a la edad en la que los padres creen que sus niños deben adquirir ciertas habilidades, y reflejan dominio de conocimientos acerca del desarrollo infantil; y 2) los comportamientos de los padres en respuesta a conductas específicas de sus hijos para ejercer control sobre ellos (prácticas disciplinarias, por ejemplo: Yo le grito a mi niño/a cuando tira la comida).

Parece conveniente aclarar que la vaga etiqueta de problemas de conducta considerada en este estudio no permitió especificar u operacionalizar los comportamientos de los niños. Pero es conveniente también mencionar que esos comportamientos se referían a señalamientos del personal de las instituciones, tales como: el niño no se está quieto, molesta a otros niños, no presta atención, etc. Por lo que evidentemente no había homogeneidad de conductas en los niños incluidos. Sin embargo, es importante señalar que las descripciones asignadas a los niños por el personal reflejaban un reto real para el manejo de los niños por ese personal.

Finalmente, entre las fortalezas de este estudio se puede argumentar que los resultados se agregan a los esfuerzos por aclarar qué factores de influencia en la crianza pueden ser modificados exitosamente por programas de intervención, como el aquí utilizado (Degnan et al., 2010), y aportan la identificación de componentes específicos que pueden ser incluidos en otros programas. Entre las debilidades de este estudio, creemos importante mencionar la ausencia de observaciones en vivo de los efectos en la socialización de los niños pequeños y de control de las variables socioeconómicas. Futuros estudios pueden tomar en cuenta estas limitaciones y creemos que pueden enriquecerse al incluir el análisis de diferentes tipos de disciplina, como pueden ser la severa y la correccional, pero sobre todo proponemos el seguimiento a largo plazo del impacto preventivo de las intervenciones para padres sobre los problemas de comportamiento de los niños preescolares.

\section{Referencias}

Abidin, R. R. (1995). Parenting Stress Index ( $3^{\mathrm{a}}$ ed.). Odessa, Florida: Psychological Assessment Resources.

Barlow, J., Coren, E. \& Stewart-Brown, S. (2003). Parent-training programmes for improving maternal psychosocial health. Cochrane Database of Systematic Reviews, 4. doi:10.1002/14651858. CD002020.pub2

Berganza, C. E., Cazali, L., Gaitán, I. \& Mezzich, J. E. (1996). Validez de criterio de la Escala de Salud del Niño. Revista Latinoamericana de Psicología, 28(2), 317-339. Recuperado de http://www. redalyc.org/pdf/805/80528208.pdf

Bongers, I. L., Koot, H. M., Van der Ende, E. J. \& Verhulst, F. C. (2003). The normative development of child and adolescent problem behavior. Journal of Abnormal Psychology, 112(2), 179-192. Recuperado de http://dx.doi.org/10.1037/0021-843X.112.2.179

Burns, G. L. \& Patterson, D. R. (2000). Factor structure of the Eyberg Child Behavior Inventory: A parent rating scale of oppositional deviant behavior toward adults, inattentive behavior, and conduct problem behavior. Journal of Clinical Child Psychology, 29(4), 569-577. doi:10.1207/S15374424JCCP2904_9

Cardona, P. G., Nicholson, B. C. \& Fox, R. A. (2000). Parenting among Hispanic and Anglo-American mothers with young children. The Journal of Social Psychology, 140(3), 357-365. doi:10.1080/00224540009600476

Conger, R. D., McCarty, J. A., Yang, R. K., Lahey, B. B. \& Kropp, J. P. (1984). Perception of child, child-rearing values, and emotional distress as mediating links between environmental stressors and observed maternal behavior. Child Development, 55(6), 22342242. doi: $10.2307 / 1129795$

Degnan, K. A., Almas, A. N. \& Fox, N. A. (2010). Temperament and the environment in the etiology of childhood anxiety. Journal of Child Psychology and Psychiatry, 51(4), 497-517. doi:10.1111/ j.1469-7610.2010.02228.x

Dishion, T. J. \& Patterson, G. R. (1992). Age effects in parent training outcome. Behavior Therapy, 23(4), 719-729. doi:10.1016/ S0005-7894(05)80231-X

Ertem, I. O., Atay, G., Dogan, D. G., Bayhan, A., Bingoler, B. E., Gok, C. G., Ozbas, S., Haznedaroglu, D. S. \& Isiklis, S. (2007). Mothers' knowledge of young child development in a developing country. Child: Care, Health and Development, 33(6), 728-737.

Eyberg, S. M., Boggs, S. R. \& Rodriguez, C. M. (1992). Relationships between maternal parenting stress and child disruptive behavior. Child and Family Behavior Therapy, 14(4), 1-9. Recuperado de https://libres.uncg.edu/ir/uncg/f/C_Rodriguez_Relationships_1992.pdf

Eyberg, S. M. \& Ross, A. W. (1978). Assessment of child behavior problems: The validation of a new inventory. Journal of Clinical Child Psychology, 7(2), 113-116. doi:10.1080/15374417809532835

Fetsch, R. J., Yang, R. K. \& Pettit, M. J. (2008). The RETHINK parenting and anger management program: A follow-up validation study. Family Relations, 57, 543-552. doi:10.1111/j.17413729.2008.00521.x

Fox, R. A., Anderson, R. C., Fox, T. A. \& Rodriguez, M. A. (1991). STAR parenting: A program for helping parents effectively deal with behavioral difficulties. Young Children, 46, 54-60. doi:10.2466/PR0.68.1.35-40

Fox, R. A. \& Fox, T. A. (1992). Leader's guide: STAR parenting program. Bellevue, Washington: STAR Parenting.

Fulton, A., Murphy, K. \& Anderson, S. L. (1991). Increasing adolescent mothers' knowledge of child development: An intervention program. Adolescence, 26(101), 73-81.

Grantham-McGregor, S. M., Cheung, Y. B., Cueto, S., Glewwe, P., Ritcher, L., Strupp, B. \& International Child Development 


\section{INTERVENCIÓN PARA PADRES}

Steering Group. (2007). Developmental potential in the first 5 years for children in developing countries. Lancet, 369, 60-70. Recuperado de http://www.thelancet.com/journals/lancet/article/ PIIS0140-6736(07)60032-4/fulltext

Hansen, K. \& Jones, E. M. (2010). Age 5 cognitive development in England. Child Indicators Research, 3(1), 105-126. doi:10.1007/ s12187-009-9055-5

Izzedin, R. \& Pachajoa, A. (2009). Pautas, prácticas y creencias acerca de crianza... ayer y hoy. Liberabit, 15(2), 109-115. Recuperado de http://www.scielo.org.pe/pdf/liber/v15n2/a05v15n2.pdf

Karoly, L. A., Greenwood, P. W., Everingham, S. S., Hoube, J., Kilburn, M. R., Rydell, C. P., Sanders, M. \& Chiesa, J. (1998). Investing in Our Children: What We Know and Don't Know about the Costs and Benefits of Early Childhood Interventions. Santa Monica, California: RAND.

Levac, A. M., McCay, E., Merka, P. \& Reddon-D'Arcy, M. L. (2008). Exploring parent participation in a parent training program for children's aggression: Understanding and illuminating mechanisms of change. Journal of Child and Adolescent Psychiatric Nursing, 21, 78-88. doi:10.1111/j.1744-6171.2008.00135.x

Lochman, J. E. \& Wells, K. C. (2003). Effectiveness study of coping power and classroom intervention with aggressive children: Outcomes at a one-year follow up. Behavior Therapy, 34(4), 493-515.

Maiuro, R. D., Vitaliano, P. P. \& Cahn, T. S. (1987). A brief measure for the assessment of anger and aggression. Journal of Interpersonal Violence, 2(2), 166-178. doi:10.1177/088626087002002003

Nicholson, B., Anderson, M., Fox, R. \& Brenner, V. (2002). One family at a time: A prevention program for at-risk parents. Journal of Counseling and Development, 80(3), 362-372. doi. org/10.1002/j.1556-6678.2002.tb00201.x

Nicholson, B. C., Brenner, V. \& Fox, R. A. (1999). A communitybased parenting program with low-income parents of young children. Families in Society: The Journal of Contemporary Human Services, 80(3), 247-253. doi:10.1606/1044-3894.678

Nicholson, B. C., Janz, P. A. \& Fox, R. A. (1998). Evaluating a brief parental-education program for parents of young children. Psychological Reports, 82, 1107-1113. doi:10.1080/0300443991480101

Ostberg, M. \& Hagekull, B. (2000). A structural modeling approach to the understanding of parenting stress. Journal of Clinical Child Psychology, 29(4), 615-625. doi:10.1207/S15374424JCCP2904_13

Sanders, M. R., Markie-Dadds, C. \& Turner, K. M. (2003). Theoretical, scientific and clinical foundations of the Triple-P Positive Parenting Program: A population approach to the promotion of parenting competence. Parenting Research and Practice Monograph, 1, 1-12.

Scott, S., Sylva, K., Moira, D., Price, J., Jacobs, B., Crook, C. \& Landau, S. (2010). Randomised controlled trial of parent groups for child antisocial behaviour targeting multiple risk factors: the SPOKES project. Journal of Child Psychology and Psychiatry, 51(1), 48-57. doi:10.1111/j.1469-7610.2009.02127.x

Silva, J. M. \& Randall, A. (2005). Giving psychology away: Educating adults to ACT against early childhood violence. Journal of Early Childhood and Infant Psychology, 1, 37-43.

Smyth, E., Whelan, T. C., McCoy, S., Quail, A. \& Doyle, E. (2010). Understanding parental influence on educational outcomes among 9 year olds in Ireland: The mediating role of resources, attitudes and children's own perspectives. Child Indicators Research, 3, 85-104. doi:10.1007/s12187-009-9051-9

Sociedad Mexicana de Psicología (2007). Código Ético del Psicólogo. México: Editorial Trillas.

Solís-Cámara, P. (2007). Manual y Escala de Comportamientos de Madres y Padres con Niños Pequeños. Instrumento, perfiles y plantillas, para computadora. Bogotá: Psicom Editores. Recuperado de http://biblomedia.com/producto/escala-comportamientos-madrespadres-con-ninos-pequenos-ecmp/

Solís-Cámara, P., Covarrubias, P., Díaz, M. \& Rivera, B. (2004). Efectos multidimensionales de un programa de crianza en la interacción recíproca de padres y sus niños con problemas de comportamiento. Psicología Conductual, 12(2), 197-214.

Solís-Cámara, P. \& Díaz, M. (2002). Efectos de un programa de crianza para mamás y papás de niños pequeños: la importancia del nivel educativo de los padres. Revista Latinoamericana de Psicología, 34(3), 203-215. Recuperado de http://www.redalyc. org/pdf/805/80534301.pdf

Solís-Cámara, P. \& Díaz, M. (2003). Expectativas del desarrollo y prácticas disciplinarias y de crianza en parejas con niños con necesidades especiales. Salud Mental, 26(2), 51-58. Recuperado de http://www.redalyc.org/pdf/582/58222606.pdf

Solís-Cámara, P., Díaz, M., Fox, R. A. \& Fox, T. (2001). Paternidad Estrella: guía de líderes-extensa. Guadalajara: Paternidad Estrella, Inc.

Solís-Cámara, P., Díaz, M., Fox, R. A. \& Fox, T. (2002). Libro de Trabajo: Paternidad Estrella. Un enfoque positivo y práctico para la paternidad eficiente ( 3 a 5 años) (2a. ed.). Guadalajara: Paternidad Estrella, Inc.

Solís-Camara, P., Fox, R. A. \& Nicholson, B. C. (2000). Parenting young children: Comparison of a psychoeducational program in Mexico and the United States. Early Child Development and Care, 163, 115-124. doi:10.1080/0300443001630108

Weymouth, L. A. (2010). Act against violence: A multi-site evaluation of the parents raising safe kids program. (Tesis de maestría inédita). California State University, Humboldt Campus, California. 
